\title{
Full replica symmetry breaking in generalized mean-field spin glasses with reflection symmetry
}

\author{
T.I.Schelkacheva, E.E.Tareyeva, and N.M.Chtchelkachev \\ Institute for High Pressure Physics, Russian Academy of Sciences, Troitsk 142190, \\ Moscow region, Russia
}

\begin{abstract}
The analysis of the solution with full replica symmetry breaking in the vicinity of $T_{c}$ of a general spin glass model with reflection symmetry is performed. The leading term in the order parameter function expansion is obtained. Parisi equation for the model is written.
\end{abstract}

Key words: Disordered systems, glass transition, replica approach, order parameter

PACS: 64.70.-p, 64.70.Kb

The crucial role of the reflection symmetry for the character of phase transition in nonrandom mean-field (MF) models is well known (see, e.g., the textbook [1]). Generally speaking the presence of the terms without reflection symmetry causes the first order phase transition, while in the absence of such terms the transition is of the second order. Usually this result is obtained in the frame of the phenomenological approach based on the Ginzburg-Landau (GL) effective Hamiltonian that can be easily obtained for any Hamiltonian through the Hubbard-Stratanovich identity for the partition function.

In the case of random MF models the behavior of systems with reflection symmetry is quite different from those without it. In Ref. [2] this problem was investigated in the frame of replica symmetric (RS) approach. The role of the reflection symmetry for the behavior of RS solution for spin-glass-like MF systems was considered and a kind of symmetry rule for the type of the growing of glass order was formulated: if in the nonrandom (pure) system the transition to the ordered phase is of the second order, then in the corresponding random system the glass regime appears as a result of a phase transition; if the transition in the pure system is of the first order, then in the random 
system the glass order parameter grows continuously on cooling. In fact, one can imagine that both the first order phase transition in nonrandom systems as well as the continuous growing of the glass order in random systems are caused by some kind of internal fields appearing due to the algebra of operators $\hat{U}$. In this case we have a close analogy with Sherrington-Kirkpatrick (SK) model in an external field.

The scenarios of replica symmetry breaking (RSB) for the mentioned two cases differ essentially one from another because the absence of reflection symmetry results in a special form of RSB free energy functional for random case, too. So, the absence of reflection symmetry can lead to continuous growing of the Edwards-Anderson order parameter as well as to a jump at the transition to one stage replica symmetry breaking (1RSB) phase and other specific features (see, for example, Refs. $[3,4,5,6]$ ). In the reflection symmetrical random systems the glass regime appears as a result of a bifurcation from the trivial solution for the order-parameter function. The prototype model is the well known Sherrington-Kirkpatrick model [7], the fully connected Ising spin model with quenched random exchange magnetic interactions. This model is described by a full replica symmetry breaking (FRSB) Parisi solution $[8,9,6,10]$.

Although the features of SK model have been thoroughly investigated, comparatively little attention has been paid to the analysis of natural generalizations of SK model (for example the systems of higher spins). So, we believe that it would be quite useful to consider a generalized spin glass model where Ising spins are replaced by arbitrary diagonal operators $\hat{U}$ with $\operatorname{Tr} \hat{U}=0$. In $\operatorname{RS}$ approximation this model was considered in [2]. In the present paper we continue the investigation of this model and consider in detail replica symmetry breaking in the case when the system possesses reflection symmetry.

Let us consider a system of particles on lattice sites $i, j$ with Hamiltonian

$$
H=-\frac{1}{2} \sum_{i \neq j} J_{i j} \hat{U}_{i} \hat{U}_{j}
$$

where $\hat{U}$ is an arbitrary diagonal operator with $\operatorname{Tr} \hat{U}=0, J_{i j}$ are quenched Gaussian interactions with zero mean

$$
P\left(J_{i j}\right)=\frac{1}{\sqrt{2 \pi J}} \exp \left[-\frac{\left(J_{i j}\right)^{2}}{2 J^{2}}\right]
$$

with $J=\tilde{J} / \sqrt{N}$.

Using replica approach (see, e.g. [7]) we can write the free energy averaged over disorder in the form [2] 


$$
\begin{aligned}
& \langle F\rangle_{J} / N k T=\lim _{n \rightarrow 0} \frac{1}{n} \max \left\{\frac{t^{2}}{4} \sum_{\alpha}\left(p^{\alpha}\right)^{2}+\frac{t^{2}}{2} \sum_{\alpha>\beta}\left(q^{\alpha \beta}\right)^{2}-\right. \\
& \left.-\ln \operatorname{Tr}_{\left\{U^{\alpha}\right\}} \exp \left[\frac{t^{2}}{2} \sum_{\alpha} p^{\alpha}\left(\hat{U}^{\alpha}\right)^{2}+t^{2} \sum_{\alpha>\beta} q^{\alpha \beta} \hat{U}^{\alpha} \hat{U}^{\beta}\right]\right\} .
\end{aligned}
$$

Here $t=\tilde{J} / k T$. The saddle point conditions for the free energy give the glass order parameter

$$
q^{\alpha \beta}=\frac{\operatorname{Tr}\left[\hat{U}^{\alpha} \hat{U}^{\beta} \exp (\hat{\theta})\right]}{\operatorname{Tr}[\exp (\hat{\theta})]}
$$

and auxiliary order parameter

$$
p^{\alpha}=\frac{\operatorname{Tr}\left[\left(\hat{U}^{\alpha}\right)^{2} \exp (\hat{\theta})\right]}{\operatorname{Tr}[\exp (\hat{\theta})]}
$$

Here

$$
\hat{\theta}=\frac{t^{2}}{2} \sum_{\alpha} p^{\alpha}\left(\hat{U}^{\alpha}\right)^{2}+t^{2} \sum_{\alpha>\beta} q^{\alpha \beta} \hat{U}^{\alpha} \hat{U}^{\beta}
$$

As one index quantity are usually replica invariant (see e.g. [5] and [6]) we put $p^{\alpha}=p$ from the very beginning.

In this paper we study the model (1) for operators $\hat{U}$ with zero trace for all odd powers:

$$
\operatorname{Tr}\left[\hat{U}^{(2 k+1)}\right]=0
$$

for all integer $k$. In the replica symmetric (RS) approximation the free energy (3) has the form:

$$
F=-N k T\left\{t^{2} \frac{q^{2}}{4}-t^{2} \frac{p^{2}}{4}+\int_{-\infty}^{\infty} \frac{d z}{\sqrt{2 \pi}} \exp \left(-\frac{z^{2}}{2}\right) \ln \operatorname{Tr}[\exp (\hat{\theta})]\right\} .
$$

Here

$$
\hat{\theta}_{R S}=z t \sqrt{q_{R S}} \hat{U}+t^{2} \frac{p-q_{R S}}{2} \hat{U}^{2}
$$

The extremum conditions for the free energy (8) give the following equations for the glass order parameter:

$$
q_{R S}=\int d z^{G}\left\{\frac{\operatorname{Tr}\left[\hat{U} \exp \left(\hat{\theta}_{R S}\right)\right]}{\operatorname{Tr}\left[\exp \left(\hat{\theta}_{R S}\right)\right]}\right\}^{2}
$$

and an auxiliary equation

$$
p=\int d z^{G} \frac{\operatorname{Tr}\left[\hat{U}^{2} \exp \left(\hat{\theta}_{R S}\right)\right]}{\operatorname{Tr}\left[\exp \left(\hat{\theta}_{R S}\right)\right]}
$$


Here

$$
\int d z^{G}=\int_{-\infty}^{\infty} \frac{d z}{\sqrt{2 \pi}} \exp \left(-\frac{z^{2}}{2}\right)
$$

If the condition (7) is fulfilled then Eq. (9) has a trivial solution for the glass order parameter $q_{R S}=0$ at any temperature. The nontrivial solution appears at the point $T_{c}$ defined by the bifurcation condition for the Eq. (9):

$$
1-t_{c}^{2} p\left(t_{c}\right)=0
$$

The replica symmetric solution is stable unless the replicon mode energy $\lambda$ is nonzero [11]. For our model (1)- (3) we have:

$$
\lambda_{r e p l(R S)}=1-t^{2} \int d z^{G}\left\{\frac{\operatorname{Tr}\left[\hat{U}^{2} \exp \left(\hat{\theta}_{R S}\right)\right]}{\operatorname{Tr}\left[\exp \left(\hat{\theta}_{R S}\right)\right]}-\left[\frac{\operatorname{Tr}\left[\hat{U} \exp \left(\hat{\theta}_{R S}\right)\right]}{\operatorname{Tr}\left[\exp \left(\hat{\theta}_{R S}\right)\right]}\right]^{2}\right\}^{2}
$$

In our case $(7)$ the condition $\lambda_{\operatorname{repl}(R S)}=0$ is equal to the equation (11) due to the fact that it is the trivial solution that bifurcates. Performing one stage replica symmety breaking (1RSB), then $2 \mathrm{RSB}, 3 \mathrm{RSB}$ etc. we can see that the r.h.s. of the equations for the glass order parameters always contains $\frac{\operatorname{Tr}\left[\hat{U} \exp \left(\hat{\theta}_{n R S B}\right)\right]}{\operatorname{Tr}\left[\exp \left(\hat{\theta}_{n R S B}\right)\right]}$. Therefore at each stage of RSB one possible solution is a trivial one and we can consider the appearance of nRSB solution as the bifurcation from the trivial (nRSB) solution. In such a case the equation $\lambda_{(n R S B)}=0$ coincides with the corresponding bifurcation condition (11). For example, in the case of $2 \mathrm{RSB}$ we have

$$
\begin{gathered}
t^{2} \int d z^{G} \frac{1}{\int d x^{G}\left(\operatorname{Tr}\left[\exp \hat{\theta}_{1 R S B}(z, x)\right]\right)^{m}} \int d y^{G}\left(\operatorname{Tr}\left[\exp \hat{\theta}_{1 R S B}(z, y)\right]\right)^{m} \\
\left\{\frac{\operatorname{Tr}\left[\hat{U}^{2} \exp \hat{\theta}_{1 R S B}(z, y)\right]}{\operatorname{Tr}\left[\exp \hat{\theta}_{1 R S B}(z, y)\right]}-\left[\frac{\operatorname{Tr}\left[\hat{U} \exp \hat{\theta}_{1 R S B}(z, y)\right]}{\operatorname{Tr}\left[\exp \hat{\theta}_{1 R S B}(z, y)\right]}\right]^{2}\right.
\end{gathered}
$$

Here

$$
\hat{\theta}_{1 R S B}(z, x)=(\sqrt{r} t z+\sqrt{v} t x) \hat{U}+\frac{t^{2}}{2}(p-r-v) \hat{U}^{2} .
$$

The 1RSB solution is characterized by $m$ and two values $r$ and $r+v$ of $q^{\alpha \beta}$. For $r=0$ and $v=0$ the equation (13) reduces to Eq. (11).

This means that the RSB solutions of different stages at least may exist at temperature $T<T_{c}$ defined by the condition (11) and encourage us to look for the full replica symmetry breaking (FRSB) $[8,9]$.

As an example let us consider the Hamiltonian (1) with $\hat{U}=\hat{Q}+\eta \hat{V}, \hat{Q}=$ $3 \hat{J}_{z}{ }^{2}-J(J+1), \hat{V}=\sqrt{3}\left(\hat{J}_{x}{ }^{2}-\hat{J}_{y}{ }^{2}\right)$. The model describes random quadrupole 


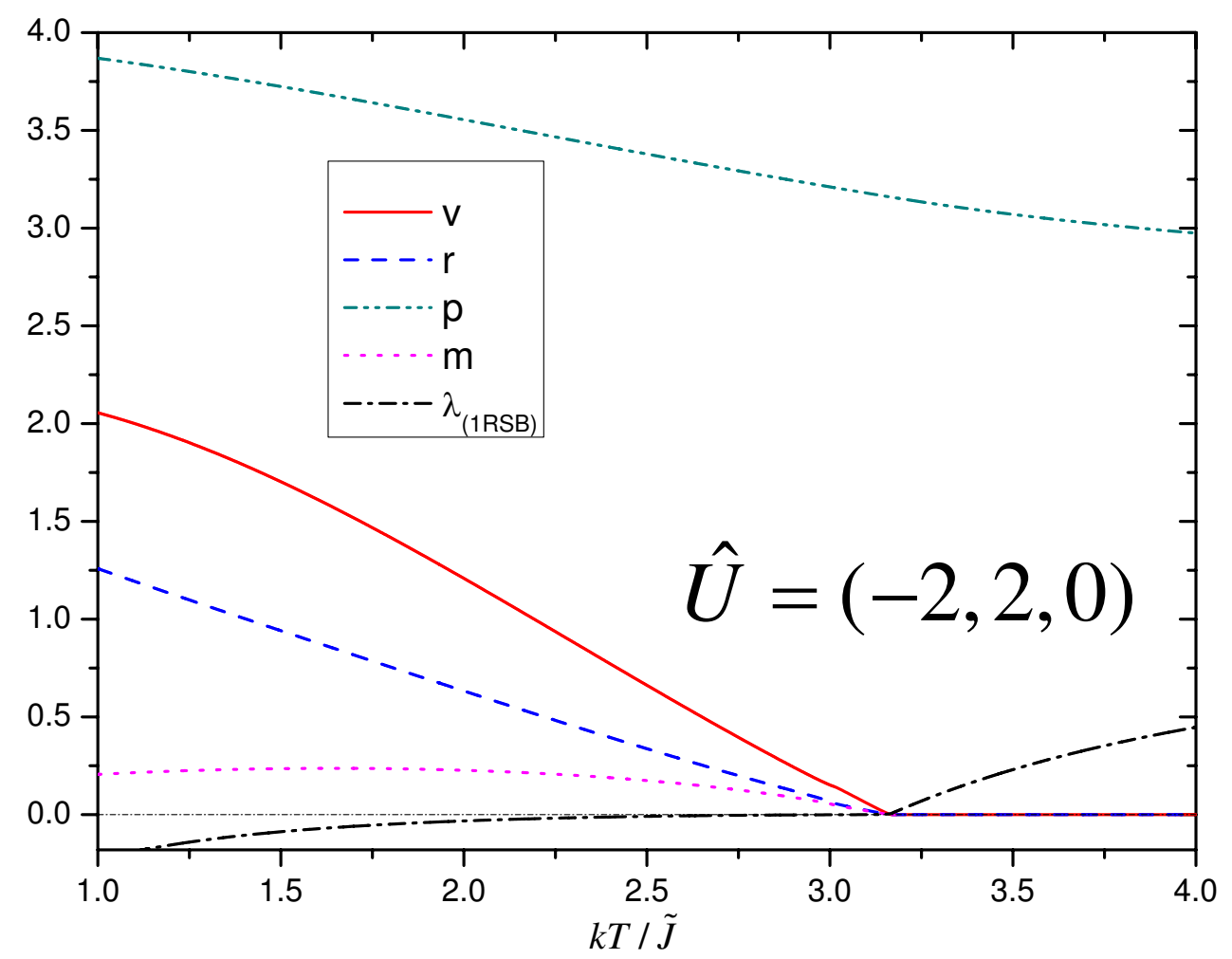

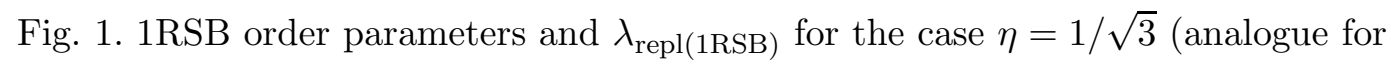
spin-one glass model). Curves for $p_{\mathrm{RS}}$ and $p_{1 \mathrm{RSB}}$ coincide.

interaction. This model has been considered for $J=1, J_{z}=1,0,-1$ in Ref. [2] and for $J=2$ in [12] in RS approximation. Here we restrict ourselves to the case $J=1$. It easy to show that $\hat{Q}^{2}=2-\hat{Q}, \hat{V}^{2}=2+\hat{Q}, \hat{Q} \hat{V}=\hat{V} \hat{Q}=\hat{V}$ and

$$
\begin{gathered}
(\hat{Q}+\eta \hat{V})^{2}=2\left(1+\eta^{2}\right)+2 \eta \hat{V}-\left(1-\eta^{2}\right) \hat{Q} \\
\left.(\hat{Q}+\eta \hat{V})^{3}=2\left(3 \eta^{2}-1\right)+3\left(1+\eta^{2}\right)(\hat{Q}+\eta \hat{V})\right] \\
\operatorname{Tr}(\hat{Q}+\eta \hat{V})^{3}=6\left(3 \eta^{2}-1\right) .
\end{gathered}
$$

Here $\eta>0$ is a tuning parameter. The behavior of the system is quite different in the cases $\eta=1 / \sqrt{3}$ (analog of spin-one glass model [15]) and $\eta \neq 1 / \sqrt{3}$.

In figures we present the behavior of the 1RSB order parameters and $\lambda_{\text {repl }(1 R S B)}$ for this case $(\eta=1 / \sqrt{3})$ and for the spin-two glass model.

In general case in FRSB scheme the series expansion in small $\delta q^{\alpha \beta}$ (up to fourth order) of the free energy (3) in the vicinity of $T_{c}$ has the following 


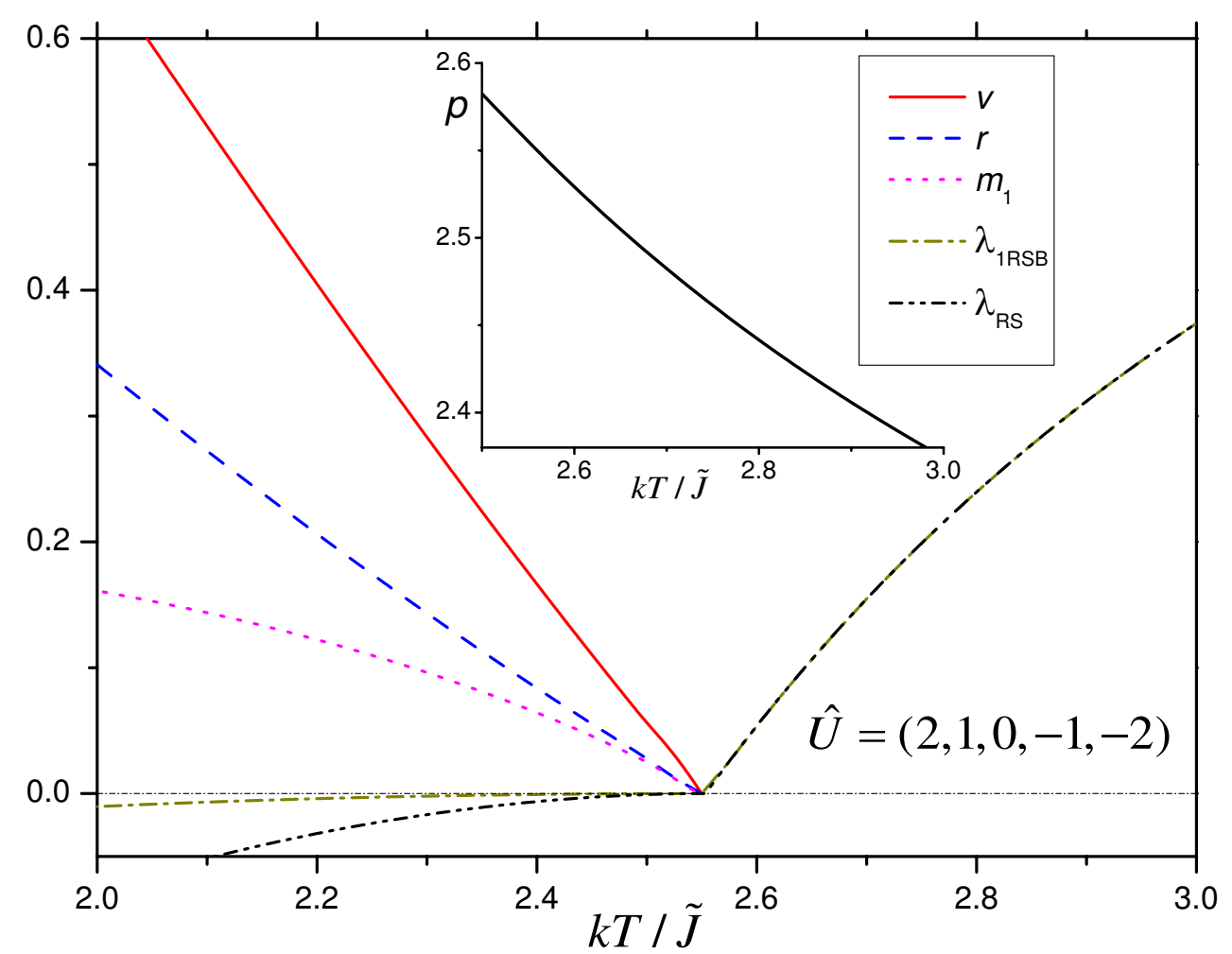

Fig. 2. 1RSB order parameters, $\lambda_{\text {repl(RS) }}$ and $\lambda_{\text {repl(1RSB) }}$ for spin-2 glass model. Curves for $p_{\mathrm{RS}}$ and $p_{1 \mathrm{RSB}}$ coincide.

form:

$$
\begin{aligned}
& \frac{\Delta F}{N k T}=\lim _{n \rightarrow 0} \frac{1}{n}\left\{\frac{t^{2}}{4} \sum\left(\delta q^{\alpha \beta}\right)^{2}-\frac{t^{4}}{4} \sum\left(\delta q^{\alpha \beta}\right)^{2}\left\langle\left[U^{2}\right]\right\rangle^{2}-\right. \\
& \frac{t^{6}}{6} \sum \delta q^{\alpha \beta} \delta q^{\beta \gamma} \delta q^{\gamma \alpha}\left\langle\left[U^{2}\right]\right\rangle^{3}- \\
& \frac{t^{8}}{8} \sum\left(\delta q^{\alpha \beta}\right)^{4} {\left[\frac{3}{2}\left\langle\left[U^{2}\right]\right\rangle^{4}-\left\langle\left[U^{4}\right]\right\rangle\left\langle\left[U^{2}\right]\right\rangle^{2}+\frac{1}{6}\left\langle\left[U^{4}\right]\right\rangle^{2}\right]-} \\
& \frac{t^{8}}{8} \sum \delta q^{\alpha \beta} \delta q^{\beta \gamma} \delta q^{\gamma \delta} \delta q^{\delta \alpha}\left\langle\left[U^{2}\right]\right\rangle^{4}+ \\
&\left.\frac{t^{8}}{8} \sum\left(\delta q^{\alpha \beta}\right)^{2}\left(\delta q^{\alpha \gamma}\right)^{2}\left[3\left\langle\left[U^{2}\right]\right\rangle^{4}-\left\langle\left[U^{4}\right]\right\rangle\left\langle\left[U^{2}\right]\right\rangle^{2}\right]\right\}
\end{aligned}
$$

Here

$$
\hat{\theta}_{0}=\frac{t^{2}}{2} p_{c} \sum\left(\hat{U}^{\alpha}\right)^{2}
$$

we use the notation

$$
\left(\frac{\operatorname{Tr} \hat{U}^{l} \exp \hat{\theta}_{0}}{\operatorname{Tr} \exp \hat{\theta}_{0}}\right)=\left\langle\left[U^{l}\right]\right\rangle
$$

and

$$
\hat{\theta}_{0}=\frac{t^{2}}{2} p_{c} \sum\left(\hat{U}^{\alpha}\right)^{2}
$$


The indices in (15) are different only if they are connected through $\delta q^{\alpha \beta}$. We believe that the parameter $p$ (5) changes slowly and is equal to $p_{c}$ in the vicinity of $t_{c}$.

Now using Parisi rules [8,9] we can write the free energy (15) in the continuous case of FRSB in the form:

$$
\begin{aligned}
& \frac{\Delta F}{N k T}= \frac{t^{4}}{4}\left\langle\left[U^{2}\right]\right\rangle^{2}\left\langle q^{2}\right\rangle\left(2 \tau-\tau^{2}\right)- \\
& \frac{t^{6}}{6}\left\langle\left[U^{2}\right]\right\rangle^{3}\left[\int_{0}^{1} d x x q^{3}(x)+3 \int_{0}^{1} d x q^{2}(x) \int_{x}^{1} q(y) d y\right]+ \\
& \frac{t^{8}}{48}\left[3\left\langle\left[U^{2}\right]\right\rangle^{2}-\left\langle\left[U^{4}\right]\right\rangle\right]^{2}\left\langle q^{4}\right\rangle-\frac{t^{8}}{8}\left\langle\left[U^{2}\right]\right\rangle^{4}\left[\left\langle q^{2}\right\rangle^{2}-4\left\langle q^{2}\right\rangle\langle q\rangle^{2}-\right. \\
&-4\langle q\rangle \int_{0}^{1} d x q(x) \int_{0}^{x}(q(y)-q(x))^{2} d y- \\
&\left.\int_{0}^{1} d x \int_{0}^{x} d y \int_{0}^{x} d z(q(x)-q(y))^{2}(q(x)-q(z))^{2}\right]- \\
& \frac{t^{8}}{8}\left\langle\left[U^{2}\right]\right\rangle^{2}\left(3\left\langle\left[U^{2}\right]\right\rangle^{2}-\left\langle\left[U^{4}\right]\right\rangle\right)\left[\left\langle q^{4}\right\rangle-\right. \\
&\left.2\left\langle q^{2}\right\rangle^{2}-\int_{0}^{1} d x \int_{0}^{x} d y\left(q^{2}(x)-q^{2}(y)\right)^{2}\right]+\ldots
\end{aligned}
$$

where

$$
\left\langle q^{l}\right\rangle=\int_{0}^{1} d x q^{l}(x), \quad \tau=1-\frac{T}{T_{c}}, \quad \frac{k T_{c}}{\tilde{J}}=p\left(T_{c}\right) .
$$

The usual approach to find the extremum of the free energy with variation with respect to $q(x)$ and further differentiations with respect to $x$ leads in the main approximation to

$$
q(x)= \begin{cases}c x, & 0 \leq x \leq \frac{\tau}{c t_{c}} \\ q(x)=\frac{\tau}{t_{c}}, & \frac{\tau}{c t_{c}} \leq x \leq 1\end{cases}
$$

with

$$
c=\frac{2\left\langle\left[U^{2}\right]\right\rangle^{5}}{\left[3\left\langle\left[U^{2}\right]\right\rangle^{2}-\left\langle\left[U^{4}\right]\right\rangle\right]^{2}}
$$

and $<q>=\tau / t_{c}$.

In close analogy to the procedure described in $[13,14]$ one can easily obtain the expansion of the free energy and of the order parameter $q(x)$ up to higher orders. One can use the approach of Parisi equation [8] for obtaining numerical solution. In our model the free energy in an external field $h$ can be written in the form

$$
\frac{F(g, h)}{N k T}=\frac{t^{2}}{4}\left[p^{2}-\int_{0}^{1} q^{2}(x) d x\right]-\int_{-\infty}^{\infty} \frac{d y}{\sqrt{2 \pi q(0)}} \exp \left[-\frac{(y-h)^{2}}{2 q(0)}\right] \phi(0, y),
$$


where $\phi(0, y)$ is the solution at $x=0$ of the Parisi equation

$$
\dot{\phi}(x, y)=-\frac{\dot{q}(x)}{2}\left[\phi^{\prime \prime}(x, y)+x\left(\phi^{\prime}(x, y)\right)^{2}\right]
$$

with the boundary condition

$$
\phi(1, y)=\ln \operatorname{Tr}\left\{\exp \left[t y \hat{U}+\frac{t^{2}}{2}(p-q(1)) \hat{U}^{2}\right]\right\} .
$$

The derivative with respect to $x$ are denoted by overdots and the derivatives with respect to $y$ by primes. In obtaining (20) - (21) the fact that $\operatorname{Tr} \hat{U}^{(2 k+1)}=$ 0 was not used. However we assume that the FRSB solution exists and $q(0)=$ 0 .

The results (16)- (21) reduce to well known form in the case of SherringtonKirkpatrick model [7] when $p=S^{2}=1$. As another example the case of spin-one can be considered. The replica symmetric solution for this model was given in [15]. In this case $3 p=2+Q$ where $Q$ is the quadrupole moment. The order-parameter function in FRSB scheme was obtained for spin-one model in $[16](K=0)$. Our results $(17)-(18)$ can be reduced to those of [16].

Now we should like to emphasize the fact that if the Hamiltonian of a model is the sum of different Hamiltonians, each of them possessing reflection symmetry, the behavior of the system can be completely different relative to that discussed here in the case of nonzero mixed terms of third order. This can be demonstrated using the toy-model with the Hamiltonian

$$
H=-\frac{1}{2} \sum_{i \neq j} J_{i j}\left[\hat{U}_{i}^{(1)} \hat{U}_{j}^{(1)}+\hat{U}_{i}^{(2)} \hat{U}_{j}^{(2)}+\hat{U}_{i}^{(3)} \hat{U}_{j}^{(3)}\right]
$$

where $J_{i j}$ are quenched Gaussian interactions with zero mean and

$$
\hat{U^{(1)}}=\left(\begin{array}{rrr}
0 & 0 & 0 \\
0 & 1 & 0 \\
0 & 0 & -1
\end{array}\right) \quad \hat{U}^{(2)}=\left(\begin{array}{rrr}
-1 & 0 & 0 \\
0 & 0 & 0 \\
0 & 0 & 1
\end{array}\right) \quad \hat{U}^{(3)}=\left(\begin{array}{rrr}
1 & 0 & 0 \\
0 & -1 & 0 \\
0 & 0 & 0
\end{array}\right)
$$

$\operatorname{Tr}\left(\hat{U}^{(l)}\right)^{3}=0$, but $\operatorname{Tr}\left[\left(\hat{U}^{(l)}\right)^{2} \hat{U}^{(k)}\right] \neq 0$ if $l \neq k$ and this results in the appearance of the term $\sum\left(\delta q^{\alpha \beta}\right)^{3}$ in the free energy expansion [17]. The model described by the Hamiltonian (22) is isomorphic to three state Potts model and has an 1RSB solution which is stable in a certain temperature interval as was shown in [18].

To conclude, in the present paper we have shown the possibility of Parisi full replica symmetry breaking for rather general model with reflection symmetry. 
The model is a generalization of SK model. We obtain the main term of the expansion of the FRSB solution for the spin glass order parameter near $T_{c}$. The Parisi equation is also written in the general case.

Authors thank V.N.Ryzhov for helpful discussions and valuable comments.

This work was supported in part by the Russian Foundation for Basic Research (Grants No.05-02-17621 (EET) and No. 05-02-17280 (TIS and NMC) and by NWO-RFBR grant No. 04-01-89005 (047.016.001)(EET and NMC).

\section{References}

[1] L.D.Landau and E.M.Lifshitz, Statistical Physics (Course of Theoretical Physics, Volume 5), 3rd edition (Butterworth-Heinemann, 1999).

[2] N.V.Gribova, V.N.Ryzhov, T.I.Schelkacheva, E.E.Tareyeva. Phys.Letters A 315, 467 (2003).

[3] P.Goldbart, and D.Elderfield. J.Phys.C 18, L229 (1985).

[4] M.Campellone, B.Coluzzi, and G.Parisi, Phys.Rev. B58, 12081 (1998).

[5] A.Crisanti and L.Leuzzi. Phys.Rev. B70, 014409 (2004).

[6] M.Mezard, G.Parisi and M.Virasoro. Spin Glass Theory and Beyond (World Scientific, Singapore, 1987).

[7] D. Sherrington and S. Kirkpatrick, Phys. Rev. Lett. 32, 1972 (1975); S. Kirkpatrick, D. Sherrington, Phys. Rev. B17, 4384 (1978).

[8] G.Parisi. J.Phys.A 13, L115 (1980).

[9] G.Parisi. J.Phys.A 13, 1887 (1980).

[10] V.A. Moskalenko, M.I. Vladimir, S.P. Koshukar. Metod samosoglasovannogo polya $\mathrm{v}$ teorii stekolnogo sostoyaniya spinovih i kvadrupolnih stekol (Stiiza, Kishinev, 1990).

[11] J.R.L.Almeida and D.J.Thouless, J.Phys.A: Math.Gen. 11, 983 (1978).

[12] T.I.Schelkacheva. Pis'ma v ZhETF, 76, 434 (2002).

[13] A.Crisanti, T.Rizzo. Phys.Rev.E 65, 046137 (2002).

[14] A.Crisanti, L.Leuzzi, G.Parisi. J.Phys. A 35, 481 (2002).

[15] E.A.Lutchinskaia and E.E.Tareyeva Theor. Math. Phys.90, 185 (1992).

[16] F.A.da Costa, F.D. Nobre, C.S.O.Yokoi. J.Phys.A: Math.Gen. 30, 2317 (1997).

[17] E.A.Lutchinskaia and E.E.Tareyeva Theor. Math. Phys.91, 157 (1992).

[18] N.V.Gribova, V.N.Ryzhov and E.E.Tareyeva. Phys.Rev. E 68, 067103 (2003). 